\title{
FORMAL MODULAR INVARIANTS WITH APPLICATION TO BINARY
}

\author{
MODULAR COVARIANTS*
}

\author{
BY \\ MILDRED SANDERSON
}

\section{$\S 1$. Introduction.}

Consider a system of forms $f_{1}\left(x_{1}, \cdots, x_{m}\right), \cdots, f_{k}\left(x_{1}, \cdots, x_{m}\right)$, whose coefficients $a_{1}, \cdots, a_{r}$ (arranged in a definite order) are independent variables. Let $b_{1}, \cdots, b_{r}$ be the coefficients (taken in the same order) of the forms derived from $f_{1}, \cdots, f_{k}$ by applying a given linear transformation $T$ with integral coefficients. Let $F\left(a_{1}, \cdots, a_{r}\right)$ be a polynomial with integral coefficients and

$$
D\left(a_{1}, \cdots, a_{r}\right)=F\left(b_{1}, \cdots, b_{r}\right)-F\left(a_{1}, \cdots, a_{r}\right)
$$

be the polynomial in $a_{1}, \cdots, a_{r}$ with integral coefficients which is obtained from $F(b)-F(a)$ upon replacing $b_{1}, \cdots, b_{r}$ by their expressions in terms of $a_{1}, \cdots, a_{r}$ and the coefficients of $T$. In case two or more of the terms in the initial expression for $D$ had the same literal part $a_{1}^{e_{1}} \cdots a_{r}^{e_{r}}$, such terms are assumed to have been combined additively into a single term. Let $p$ be a prime. According to the definition by Hunwitzt '(stated for a single form in two variables), $F\left(a_{1}, \cdots, a_{r}\right)$ is an invariant of $f_{1}, \cdots, f_{k}$ modulo $p$ with respect to the transformation $T$ if $D\left(a_{1}, \cdots, a_{r}\right) \equiv 0(\bmod p)$, identically in $a_{1}, \cdots, a_{r}$, viz., if the coefficient of each term of $D$ is divisible by $p$. He gave an interesting example of such an invariant with respect to all of the transformations $T$ with integral coefficients whose determinant is not divisible by $p$, but did not construct a theory of invariants modulo $p$.

As a generalization we may employ transformations $T$ with coefficients in the Galois field $G F\left[p^{n}\right]$ composed of the $p^{n}$ elements

$$
c_{0}+c_{1} j+c_{2} j^{2}+\cdots+c_{n-1} j^{n-1} \quad\left(c_{0}, \cdots, c_{n-1}=0,1, \cdots, p-1\right),
$$

where $j$ is a root of a congruence of degree $n$ irreducible modulo $p$. A polynomial $F\left(a_{1}, \cdots, a_{r}\right)$ with integral coefficients is a formal invariant of $f_{1}, \cdots, f_{k}$, in the field, under transformation $T$ if $\ddagger D\left(a_{1}, \cdots, a_{r}\right)$ is identi-

* Presented to the Society (Chicago), March 21, 1913, under different title.

† Archivder Mathematik und Physik, ser. 3, vol. 5 (1903), p. 22.

$\ddagger$ In the spirit of KRONECKER's use of polynomials in indeterminates $a_{1}, \cdots, a_{r}$ with coefficients in any domain of rationality, we use polynomials with coefficients in the $G F\left[p^{n}\right]$. Cf. J. KïöNı, Theorie der algebraischen Grössen, Leipzig, 1903, p. 31; H. Werer, M a th ematische Annalen, vol. 43 (1893), p. 526. 
cally zero in the field as to $a_{1}, \cdots, a_{r}$, i. e., if the coefficient of each term of $D$, when reduced to the form $c_{0}+c_{1} j+\cdots+c_{n-1} j^{n-1}$ by means of the congruence satisfied by $j$, has each $i_{i}$ a multiple of $p$. If $F_{0}, F_{1}, \cdots$ are such formal invariants (with integral coefficients), then $F_{0}+F_{1} j+\cdots$ is a formal invariant. In this manner, or by direct extension of the previous definition, we have the concept of a formally invariant polynomial with coefficients in the $\left(i F\left[p^{n}\right]\right.$.

We pass to the entirely different concept of modular invariants, introduced by I)I(ksox.* The coefficients $a_{1}, \cdots, a_{r}$ of the forms are now undetermined elements of the $G F\left[p^{n}\right]$. A polynomial $F\left(a_{1}, \cdots, a_{r}\right)$ with coefficients in that field is called a modular invariant of the system of forms under any given group ( $i$ of linear transformations with coefficients in the field if, for each transformation of $G, I)\left(a_{1}, \cdots, a_{r}\right)$ is zero in the field. To apply this test we may first expresst $D$ as a polynomial $\delta\left(a_{1}, \cdots, a_{r}\right)$ in which the exponents are all less than $p^{n}$, and then require that $\delta$ shall be identically zero in the ficld as to $a_{1}, \cdots, a_{r}$. We thus see clearly just how the difference in the definitions of formal and modular invariants affects the actual computations. 1)ickison + has given a very simple and elegant theory of modular invariants. No theory has been developed for formal invariants. However, there exists between the two subjects an interesting and important relation, which 1 shall derelop in what follows. I take this opportunity to express my gratitude to Professor Dickson for his interest and many helpful suggestions, in particular for the present formulation of this introductory section.

\section{\$2. Statement and application of the fundamental theorem.}

To any modular invariant $i$ of a system of forms under any group) ( $A$ of linear transformations with coefficients in the $\left(i F\left[p^{n}\right]\right.$, there corresponds a formal invariant $I$ under $G$ such that $I=i$ for all sets of values in the field of the corfficients of the system of forms.

This theorem enables us, as in the algebraic theory of invariants, to construct covariants of a system of binary forms in $x$ and $y$ from invariants of this system and an additional linear form whose coefficients are $y$ and $-x$, if the invariants are made formally invariant with respect to $x$ and $y$. Noreover, every invariant will yield a covariant. Some invariants will yield two or more independent covariants. For example,

$$
f=a_{0} x^{2}+2 a_{1} x y+a_{2} y^{2}, \quad a_{0} x^{4}+a_{1} x^{3} y+a_{1} x y^{3}+a_{2} y^{4}
$$

* These Transactions, vol. 8 (1907), p. 205.

$\dagger \mathrm{By}$ use of Galois's generalization $a^{p^{n}}=a$ of Fermat's theorem.

† These Transactions, vol. 10 (1909), p. 123; American Journal of Mathematics, vol. 31 (1909), p. 337. 
are two independent covariants modulo 3 of $f$, but as modular invariants of $f$ and an additional linear form they are to be identified. The universal covariant

$$
\begin{cases}x & y \\ x^{p^{\prime \prime}} & y^{p^{\prime \prime}}\end{cases}
$$

on the other hand, could not be obtained as an invariant of a linear form, because it vanishes when $x$ and $y$ are in the $\left(i F\left[y^{n}\right]\right.$, as we suppose the coefficients of our forms to be. Whether or not all the covariants of a system of forms can be expressed as functions of this universal covariant and the invariants of this system and a linear form is a question as yet unanswered. In some special cases this has proved to be the case. In later sections will be shown a practical method for constructing covariants together with an illustration of the fact just mentioned. (See $\$ \$(;, 7$.

In order to prove the above theorem, we shall first establish an interesting lemma.

\section{\$3. Linear factors of a certain determinant $D$.}

Le'mma. Let $a_{1}, \cdots, a_{r}(r>1)$ be arbitrary variables, and $g_{1}, \cdots, !_{r}$ given elements of the $\left(i F\left[p^{n}\right], g_{r} \neq 0\right.$. Then the determinant

$$
N=\begin{array}{llll}
a_{1}, & \cdots, & a_{r} \\
a_{1}^{p^{\prime \prime}}, & \cdots, & a_{r}^{, n} \\
\cdot & \cdot & \cdot & \cdot \\
a_{1}^{p^{(r-1) n}}, & \cdots, & a_{r}^{p^{(r-1) n}}
\end{array}
$$

is dirisible in the ficld by the determinant

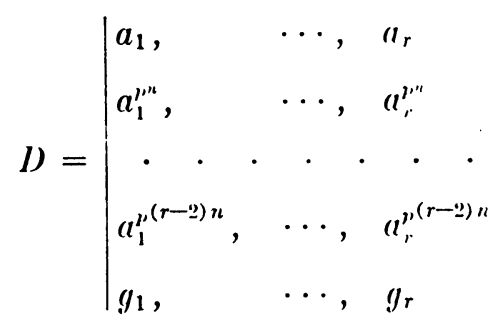

and the quoticut $(\ell=N /$ I) has the propertices

$$
\begin{aligned}
& Q \neq 0 \quad \text { if } \quad a_{1}=l_{1}, \cdots, a_{r}=g_{r}, \\
& Q=0 \text { if } a_{1}=\iota_{1}, \cdots, a_{r}=c_{r},
\end{aligned}
$$

where $e_{1}, \cdots, e_{r}$ are elements of the field not proportional to $g_{1}, \cdots, g_{r}$. 
We know that $N$ is an invariant and is equal to the following product:*

$$
N=\prod_{k=1}^{r} \prod_{b}\left(a_{k}+b_{k+1} a_{k+1}+\cdots+b_{r} a_{r}\right),
$$

where the second product extends over the $p^{(r-k) n}$ sets $b_{k+1}, \cdots, b_{r}$ obtained when each $b$ ranges independently over the elements of the $G F\left[p^{n}\right]$.

Let us consider the determinant $D$. We can see at once that it is divisible by

$$
a_{1}-\frac{g_{1}}{g_{r}} a_{r},
$$

since this divides all the two-rowed minors formed from the first and $r$ th columns. If we transform the variables by setting

$$
a_{1}=A_{1}+\sum_{j=2}^{r} k_{j} A_{j}, \quad a_{j}=A_{j}, \quad(j=2, \cdots, r),
$$

and subtract from the elements of the first column of $D$ the products of the elements of the $j$ th column $(j=2, \cdots, r)$ by $k_{j}$, we may write $D$ in the form

$$
\begin{aligned}
& A_{1}, \quad A_{2}, \quad \cdots, A_{r} \\
& D=A_{1}^{p^{(r-2) n}}, \quad A_{2}^{p(r-2) n}, \cdots, A_{r}^{p(r-2) n} . \\
& g_{1}-\sum_{j=2}^{r} k_{j} g_{j}, g_{2}, \quad \cdots, \quad g_{r}
\end{aligned}
$$

Thus $D$ will be invariant under all transformations (6) for which

$$
\sum_{j=2}^{r} k_{j} g_{j}=0 .
$$

Since $g_{r} \neq 0$, the first $r-2$ l's may be chosen arbitrarily, hence in $p^{(r-2) n}$ ways, and $k_{r}$ will be uniquely determined. If we transform (5) by all the transformations $(6)$ which satisfy $(8)$ we get $p^{(r-2) n}$ different linear factors, which upon replacing the $A_{i}$ by the $a_{i}$ are

$$
a_{1}+\sum_{j=2}^{r} k_{j} a_{j}-\frac{g_{1}}{g_{r}} a_{r} .
$$

These must divide $D$ since $D$ was invariant under all such transformations. From (\$) we see easily that all of these linear factors vanish for $a_{1}=g_{1}, \cdots$, $a_{r}=g_{r}$.

Since $D$ is also divisible by

$$
a_{2}-\frac{g_{2}}{g_{r}} a_{r}, \quad \cdots, \quad a_{r-1}-\frac{g_{r-1}}{g_{r}} a_{r},
$$

* L. E. Dickson, these Trans a ctions, vol. 12 (1911), p. 76, and references there given. 
by using transformations similar to (6), namely,

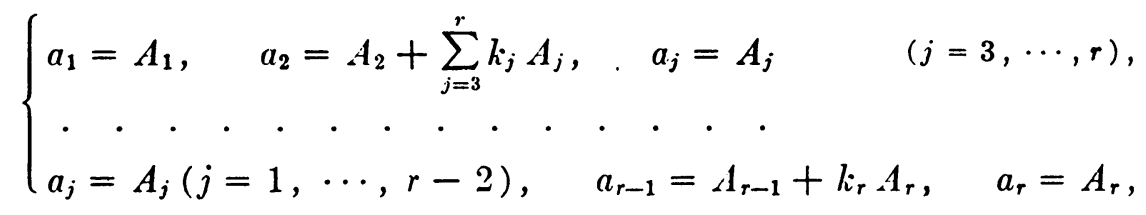

we find that $D$ is divisible by respectively

$$
p^{(r-3) n}, \quad \cdots, \quad p^{n}, \quad 1
$$

new linear factors all of which ranish for $a_{1}=g_{1}, \cdots, a_{r}=g_{r}$. These

$$
p^{(r-2) n}+p^{(r-3) n}+\cdots+p^{n}+1=w
$$

factors are all distinct. Hence $D$ is divisible by their product. But $u$ is exactly the degree of $D$, so that, apart from the factor $\pm g_{r}, D$ is equal to this product. It may be written

$$
D= \pm g_{r} \prod_{k=1}^{r} \prod_{\{b]}\left(a_{k}+b_{k+1} a_{k+1}+\cdots+b_{r} a_{r}\right)
$$

where the second product extends over all values of the $b_{j}$ in the $G F\left[p^{n}\right]$ such that the linear form vanishes for $a_{1}=g_{1}, \cdots, a_{r}=g_{r}$.

Since all these factors are contained in $N, Q$ is integral. Now $D$ contains all linear forms with unity for the coefficient of the $a_{j}$ of lowest subscript, which vanish for $a_{i}=g_{i}(i=1, \cdots, r)$. Hence, none of the factors of $Q$ vanish for $a_{i}=g_{i}$, so that $\left(\ell \neq 0\right.$ for $a_{1}=g_{1}, \cdots, a_{r}=g_{r}$.

Some of the factors of the quotient must vanish for $a_{1}=e_{1}, \cdots, a_{r}=c_{r}$, where $e_{1}, \cdots, e_{r}$ are not proportional to $g_{1}, \cdots, g_{r}$. For, there are $w$ factors of $N$ which do vanish, and unless these are the same as the factors of $D, Q$ must vanish. But $r-1$ of the factors of $D$, namely,

$$
a_{1}-\frac{g_{1}}{g_{r}} a_{r}, \quad a_{2}-\frac{g_{2}}{g_{r}} a_{r}, \quad \cdots, \quad a_{r-1}-{ }_{g_{r}}^{g_{r-1}} a_{r},
$$

are linearly independent, and hence when equated to zero uniquely determine the ratios of the $a$ 's for which $l)$ vanishes. 'Therefore, if $Q$ does not vanish for $a_{1}=e_{1}, \cdots, a_{r}=e_{r}$, then $e_{1}, \cdots, e_{r}$ must be proportional to $g_{1}, \cdots, g_{r}$.

\section{$\$ 4$. Definition of the classes $\left({ }^{r} i j\right.$.}

consider a sistem $S$ of forms $f_{1}, \cdots, f_{l}$ in $m$ variables with coefficients $a_{1}, \cdots, a_{r}$. Following the method of Professor I. E. 1)ickson, ${ }^{*}$ we shall give the coefficients of $S$ special values in the $\left(i F\left[p^{n}\right]\right.$, and divide the resulting

* These 'Transactions, vol. 10 (1909), p. 12:3; American Journal of Il a thematics, vol. $31(1909)$, p. 333 . 
set of systems into classes $C_{i}$, such that $S^{\prime}$ and $S^{\prime \prime}$ shall belong to the same class if and only if they are equivalent under $\left(r\right.$. If $S^{\prime}$ is a particular system of forms $f_{1}^{\prime}, \cdots, f_{t}^{\prime}$, and if $k$ is a constant, we shall say that the system of forms $l \cdot f_{1}^{\prime}, \cdots, l \cdot f_{t}^{\prime}$ is a multiple of $S^{\prime}$, and we shall denote it by $k S^{\prime}$. Let $c_{i}$ be a subset of $C_{i}$ such that if $s$ is in $c_{i}$ no multiple of $s$ is in $c_{i}$, and such that every system $S$ in $C_{i}$ is a multiple of some sistem in $c_{i}$. Let $\rho$ be a primitive root in the $G F\left[p^{n}\right]$. Suppose $e_{i}$ to be the smallest exponent for which $s^{\prime}$ and $\rho^{e_{i}} s^{\prime}$ are equivalent under $G^{\prime}$, where $s^{\prime}$ is any system in $c_{i}$. Then $e_{i}$ is a factor of $p^{n}-1$ and

$$
s^{\prime}, \quad \rho^{e_{i}} s^{\prime}, \quad \cdots, \quad \rho^{\left(d_{i}-1\right) e_{i}} s^{\prime} \quad\left(d_{i} e_{i}=p^{n}-1\right)
$$

are all contained in $C_{i}{ }_{i}$. If $S^{\prime \prime}$ is a system in $C_{i}$ not in set (13), $\rho^{e_{i}} S^{\prime \prime}$ will be in $C_{i}$. For, $S^{\prime \prime}$ may be transformed into $s^{\prime}, s^{\prime}$ into $\rho^{e_{i}} s^{\prime}, \rho^{e_{i}} s^{\prime}$ into $\rho^{e_{i}} S^{\prime \prime}$. Moreover any system $S^{\prime \prime \prime}$ in $C_{i}^{\prime}$ is equal to $\rho^{k e_{i}}$ times a șistem $s^{\prime \prime \prime}$ in $c_{i}$. For we may write

$$
S^{\prime \prime \prime}=\rho^{k e_{i}+l} s^{\prime \prime \prime} \quad\left(0 \leqq l<c_{i}\right) .
$$

Since $\rho^{p^{n-1-k e_{i}}} S^{\prime \prime \prime}$ is in $C_{i}, \rho^{l} s^{\prime \prime \prime}$ is in $C_{i}$. But $c_{i}$ is the smallest exponent for which this is possible. Hence $l=0$. Therefore we may write

$$
C_{i}=\sum_{i=1}^{d_{i}} c_{i} \rho^{k e_{i}},
$$

where $\Sigma$ denotes an aggregate, and $c_{i} \rho^{k e_{i}}$ is the set of systems obtained by multiplying each ș'stem in $c_{i}$ by $\rho^{k_{i}}$. If $e_{i}=1, C_{i}^{\prime}$ will contain all the multiples of the sistems in $c_{i}$. In general there are $c^{\prime}{ }_{i}$ different classes

$$
C^{\prime}{ }_{i l}=\sum_{l=1}^{l_{i}} c_{i} \rho^{k e_{i}+l} \quad\left(l=0, \cdots, c_{i}-1\right)
$$

formed on the subsets $c_{i}, c_{i} \rho, \cdots, c_{i} \rho^{e_{i}-1}$ respectively. 'Thus a complete list of the classes is given by $(14)$, where $i=1, \cdots, q$, and $C_{00}^{\prime}$ which contains only the identically zero system.

\section{§5. C'haracteristic incariants.}

All invariants of the system $S$ of forms can be expressed in terms of characteristic invariants* $i_{k l}$ which have the value one for systems of the class $C_{k l}$ and the value zero for systems in the remaining classes. To prove the theorem of $\S 2$, it suffices to construct formal invariants $I_{k l}$ which reduce for values of the coefficients in the $G F\left[p^{n}\right]$ to the characteristic invariants $i_{k l}$. For the construction of the $I_{k l}$ it is convenient to employ the invariants

$$
J_{i}=\sum_{y_{1}, \ldots, !_{r}}^{c_{i}}\left[Q\left(g_{1}, \cdots, g_{r}\right)\right]^{l_{i}} \quad(i=1, \cdots, q),
$$

* L. E. Dickson, l. c. (preceding foot-note). 
where the sum extends over the different sets of coefficients in $c_{i}$. When the variables are transformed under $G$, the coefficients $a_{1}, \cdots, a_{r}$ undergo an induced transformation which is also linear with coefficients in the $G F\left[p^{n}\right]$. $J_{i}$ is a formal invariant since the numerators are invariant apart from a factor which is the $d_{i}$ th power of the determinant of the induced transformation, and the denominators are permuted among themselves apart from the same factor.

Consider any particular denom nator

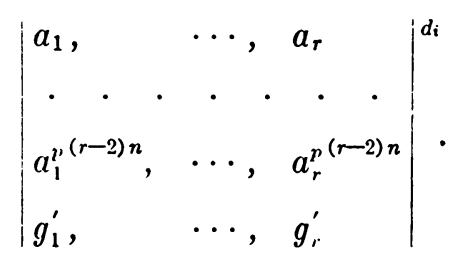

Since $g_{1}^{\prime}, \cdots, g_{r}^{\prime}$ are the coefficients of a system of $c_{i}$, this system is also in $C_{i 0}$. After transformation this will become

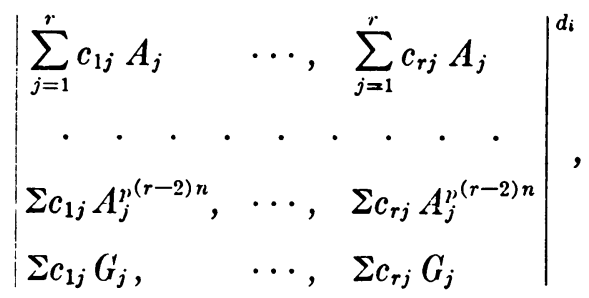

where $G_{1}, \cdots, G_{r}$ are also the coefficients of a system in $C_{i 0}$ and hence may be written $\rho^{l e_{i}} g_{1}^{\prime \prime}, \cdots, \rho^{l e_{i}} g_{r}^{\prime \prime}$, where $g_{1}^{\prime \prime}, \cdots, g_{r}^{\prime \prime}$ are the coefficients of a system in $c_{i}$. Hence (17) becomes

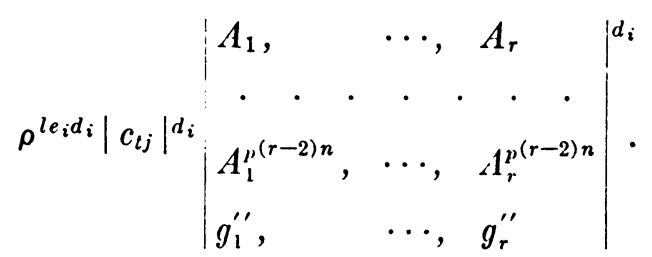

Since $\rho^{l_{i} d_{i}}=1$, this denominator apart from the factor $\left|c_{t j}\right|^{d_{i}}$ occurs. among the denominators in the sum (1ij), and the factor $\left|c_{t j}\right|^{d_{i}}$ is canceled by the same factor which is brought into the numerator by the transformation. Hence $J_{i}$ is a formal invariant.

Let $J_{i}\left(C_{j k}\right)$ denote the value which $J_{i}$ has for systems of the class $C_{j k}$. Obriously $J_{i}\left(C_{00}\right)=0$. In view of (15) and (3),

$$
J_{i}\left(C_{k l}\right)=0 \quad(k \neq i), J_{i}\left(C_{i l}\right) \neq 0 .
$$


Since $J_{i}\left(C_{i 0}\right) \neq 0$, we may set

$$
J_{i}\left(C_{i 0}\right)=\rho^{l} .
$$

We can easily see that

$$
J_{i}\left(C_{i k}\right)=\rho^{l+k d_{i}} \quad\left(k=0,1, \cdots, e_{i}-1\right) .
$$

By making use of the fact that any invariant $I$ may be written in the form*

$$
I=\sum_{j k} v_{j k} I_{j k},
$$

where $v_{j k}$ is the value which $I$ has for systems in the class $C_{j k}$, we obtain the following equations for the determination of the $I_{i k}$ :

$$
J_{i}^{\prime \prime}=\sum_{i=0}^{e_{i}-1} \rho^{g\left(l+k \prime_{i}\right)} I_{i k} \quad\left(g=1, \cdots, e_{i}\right) .
$$

The determinant of the coefficients is

$$
\left|\rho^{g\left(l+k d_{i}\right)}\right|=\rho^{l\left(1+2+\cdots+e_{i}\right)}\left|\left(\rho^{d_{i}}\right)^{g k}\right|\left(k=0, \cdots, e_{i}-1 ; g=1, \cdots, e_{i}\right) .
$$

This is not zero sipce it is equal to the product of differences of distinct powers of $\rho$. Hence we can solve (22) uniquely for the $I_{i k}$.

The characteristic invariant for the class $C_{00}$ is

$$
I_{00}=1-\sum_{g_{1}, \ldots g_{r}}^{c}\left[Q\left(g_{1}, \cdots, g_{r}\right)\right]^{p^{n}-1}
$$

where $c$ is the set of all subsets $c_{i}$.

\section{$\S 6$. Construction of binary covariants.}

Let us consider the covariants of a form $f$ of the $m$ th degree with coefficients in the $G F\left[p^{n}\right]$ under the group of all binary linear transformations with coefficients in the $G F\left[p^{n}\right]$. We may assume that it has been broken up into $m$ linear factors whose coefficients are in a Galois Field of order $p^{m ! n}$ or lower. We shall write it

$$
f=\prod_{j=1}^{m}\left(\alpha_{1}^{(j)} x_{1}+\alpha_{2}^{(j)} x_{2}\right)=\prod_{j=1}^{m} \alpha_{i}^{(j)}=\prod_{j=1}^{m} x_{a}(j) .
$$

When the variables are transformed, the coefficients of each linear form are transformed contragrediently to the variables. Any function $F$ of the $\alpha$ 's and the $x$ 's which is invariant and which is unchanged when the superscripts on any two sets of $\alpha$ 's are interchanged will be a covariant of the form $f$. If we call any two of the linear factors of $f \alpha_{x}$ and $\beta_{x}, F$ may obviously contain

\footnotetext{
* I. E. Dickson, l. c.
} 
functions of the type

$$
\begin{aligned}
& \alpha_{x}, \quad \alpha_{x} p^{n}=\alpha_{1} x_{1}^{3 n}+\alpha_{2} x_{2}^{p^{\prime n}}, \quad x_{a} p^{n}, \\
& \mid \begin{array}{ll}
\alpha_{1}^{p^{n}} & \alpha_{2}^{\nu^{n}} \\
\alpha_{1}^{p k n} & \alpha_{2}^{p^{k n}}
\end{array}=\left(\begin{array}{ll}
\alpha^{p^{j n}} & \alpha^{p^{k n}}
\end{array}\right), \\
& \left|\begin{array}{ll}
\alpha_{1}^{p n} & \alpha_{2}^{p^{n n}} \\
\beta_{1}^{p k n} & \beta_{2}^{p^{k n}}
\end{array}\right|=\left(\begin{array}{ll}
\alpha^{p^{j n}} & \beta^{p^{k n}}
\end{array}\right), \\
& \left|\begin{array}{ll}
x_{1}^{p^{n n}} & x_{2}^{p^{j n}} \\
x_{1}^{p^{k n}} & x_{2}^{p^{k n}}
\end{array}\right|=\left(\begin{array}{ll}
x^{p^{j n}} & x^{p^{k n}}
\end{array}\right) .
\end{aligned}
$$

It is not necessary that $F$ should be integral in these functions, but if the covariant is to be integral, $F$ must be integral in the $\alpha$ 's and $x$ 's .

\$7. The covariants of a binary quadratic form in the $G F\left[p^{n}\right](p>2)$.

Consider the form

$$
f=a x_{1}^{2}+2 b x_{1} x_{2}+c x_{2}^{2}=\alpha_{x} \beta_{x}
$$

with coefficients in the $G F\left[p^{n}\right]$. We wish to find the covariants of this form under the group of all binary linear transformations with coefficients in the $G F\left[p^{n}\right]$. The coefficient of the highest power of $x_{1}$ is a seminvariant, that is, it is invariant under all the transformations of the type

$$
x_{1}=x_{1}^{\prime}+\lambda x_{2}^{\prime}, \quad x_{2}=x_{2}^{\prime} .
$$

We shall first find the seminvariants of the form, and then construct covariants from these.

Under the group of transformations (28), the forms $f$ are seen to belong to one of the following classes:

$$
\left.C_{\rho^{i}, d}, \quad C_{\rho^{i}}, \quad \underset{\left(d=0, \rho, \rho^{2}\right.}{ } C, \cdots, \rho^{p^{n-1}} ; i=1, \cdots, p^{n}-1\right),
$$

where $\rho$ is a primitive root in the $G F\left[p^{n}\right]$. The canonical forms are respectively:

$$
\rho^{i}\left(x_{1}^{2}+d x_{2}^{2}\right), \quad \rho^{i} x_{1} x_{2}, \quad \rho^{i} x_{2}^{2}, \quad 0 .
$$

The classes are completely characterized by the seminvariants

$$
\begin{aligned}
D & =\frac{1}{4}(\alpha \beta)^{2}=b^{2}-a c, \\
a & =\alpha_{1} \beta_{1}, \\
R & =\left(\alpha_{1}^{n n-1} \alpha_{2}-\alpha_{2}^{p^{n}}\right)\left(\beta_{1}^{p^{n}-1} \beta_{2}-\beta_{2}^{p^{n}}\right), \\
B & =\frac{1}{2} \sum_{2}\left(\alpha^{p^{n}} \beta\right) \beta_{1}^{p^{n}-1}=a^{p^{n}-1} b-b^{p^{n}},
\end{aligned}
$$


as is seen by the accompanying table which gives the value that each seminvariant has for the respective classes. They also form a fundamental set.

\begin{tabular}{|c|c|c|c|c|}
\hline & $C_{\rho^{i}, l}$ & $C_{\rho^{i}}$ & ${ }_{\rho^{i}} C$ & $C_{0}$ \\
\hline$D$ & $-\rho^{2 i} d$ & $\rho^{2 i} i 4$ & 0 & 0 \\
\hline$a$ & $\rho^{i}$ & 0 & 0 & 0 \\
\hline$R$ & $\begin{array}{c}(-d \text { square }) 0 \\
(-d \text { not square }) 4 \rho^{i} d \\
(-d \text { zero }) 0\end{array}$ & 0 & $\rho^{i}$ & 0 \\
\hline$B$ & 0 & $-\rho^{i} / 2$ & 0 & 0 \\
\hline
\end{tabular}

All seminvariants must then be functions of these. However, there are some others which have very simple expressions, but which as functions of these four might be very complicated. They are

$$
\begin{aligned}
& B_{j}=\frac{1}{2} \sum_{2}\left(\alpha^{p^{n}} \beta\right)^{j} \beta_{1}^{j\left(p^{n}-1\right)}, \\
& S=\sum_{2} \alpha_{1}^{2 \tau p^{n}} \frac{\left(\beta \beta^{p^{2 n}}\right)}{\left(\beta \beta^{p^{n}}\right)} \quad\left(\tau=\frac{p^{n}-1}{2}\right) \text {. } \\
& q=\left(a^{\tau}+c^{\tau}\right) \sum_{i=0}^{\tau} a^{i} c^{i} b^{2 \tau-2 i}-a^{3 \tau}-c^{3 \tau} .
\end{aligned}
$$

The only invariants among these are $D$ and $q$, and these form a fundamental system of invariants.*

By comparing the values which the different seminvariants have for the different classes according to tables (32) and

\begin{tabular}{c|c|c|c|c} 
& $C_{\rho^{i}, d}$ & \multicolumn{2}{c}{$C_{\rho^{i}}$} & \multicolumn{2}{c}{${ }_{\rho^{i}} C$} & $C_{0}$ \\
\hline$q$ & $(-1)^{i}\left[d^{2 \tau}-1\right]$ & 0 & $(-1)^{i+1}$ & 0 \\
\hline$B_{j}$ & \begin{tabular}{c}
$\left(\begin{array}{c}\text { even } \\
-d \text { square } \\
(\text { otherwise }) 0\end{array} 2^{j} \rho^{j i}(-d)^{\frac{j}{2}}\right.$ \\
\hdashline
\end{tabular} & $\frac{1}{2}(-1)^{j} \rho^{j i}$ & 0 & 0 \\
\hline$S$ & $\begin{array}{c}(-d \text { square } 2 \\
(-d \text { not square) } 0 \\
(-d \text { zero) 2 }\end{array}$ & 1 & 0 & 0 \\
\hline
\end{tabular}

we find various syzygies, among which are

$$
\begin{gathered}
D^{\tau}+a^{2 \tau}=S+a^{\tau} q, \\
q=R^{\tau} a^{2 \tau}+D^{2 \tau} S^{2 \tau} a^{\tau}-S^{2 \tau} a^{\tau}-R^{\tau} .
\end{gathered}
$$

*L. E. Dickson, American Journal of Mathematics, vol. 31 (1909) p. 109; these Tra n s a c tion s, vol. 10 (1909), p. 132. 
Covariants are formed very easily from the seminvariants $a, B=B_{1}$, $B_{j}, S$ and powers of these by replacing $\alpha_{1}$ and $\beta_{1}$ by $\alpha_{x}$ and $\beta_{x}$ or $\alpha_{x} p^{n}$ and $\beta_{x} p^{n}$, and by replacing $\alpha_{1}^{p n}$ and $\beta_{1}^{p^{n}}$ by $x_{a^{\prime n}}$ and $x_{\beta^{\prime n}}$, wherever the $\alpha_{1}$ and $\beta_{1}$ do not occur in one of the invariant determinants.

Thus with $a$ as a leader we get the two distinct covariants:

$$
f=\alpha_{x} \beta_{x}, \quad f_{1}=\frac{1}{2} \sum_{2} \alpha_{x} p^{n} \beta_{x} .
$$

Covariants led by $B_{j}$, where $j<p^{n}$, are

$$
\begin{array}{ccc}
\frac{1}{2} \sum_{2}\left(\alpha^{p^{n}} \beta\right)^{j} \beta_{x}^{j\left(p^{n}-1\right)}, & \frac{1}{2} \sum_{2}\left(\alpha^{p^{n}} \beta\right)^{j} x_{\beta} p^{n} \beta_{x}^{(j-1) p^{n}-j}, \\
\cdot . \quad . \quad . \quad . \quad . ~ & \cdot & \frac{1}{2} \sum_{2}\left(\alpha^{p^{n}} \beta\right)^{j} x_{\beta}^{j-1} \beta_{x}^{p^{n}-j} .
\end{array}
$$

The seminvariant $S$ will lead the covariants:

$$
\sum_{2} x_{a}^{k} p^{n} \alpha_{x}^{(2 \tau-k) p^{n}} \frac{\left(\beta \beta^{p^{2 n}}\right)}{\left(\beta \beta^{p^{n}}\right)} \quad\left(k=0,1, \cdots, p^{n}-1\right) .
$$

A simple method has not yet been found for constructing covariants with a leading coefficient $R$.

In addition to those already mentioned, should be noted the universal covariants*

$$
L=\left(x x^{p^{n}}\right), \quad Q=\frac{\left(x x^{p^{2 n}}\right)}{\left(x x^{p^{n}}\right)} .
$$

For the general Galois Field of order $p^{n}$, a complete system of covariants of the binary quadratic form has not yet been found. For the case $p^{n}=3$ Professor L. E. Dicksont has found the complete system, but not their expressions in terms of the present symbols. The fundamental covariants are included among those mentioned above. They are

$$
\begin{aligned}
D & =(\alpha \beta)^{2}=b^{2}-a c, \\
q & =\frac{\left(\alpha^{3} \beta\right)^{2}(\alpha \beta)\left(\alpha \alpha^{3}\right)-\left(\alpha \beta^{3}\right)\left(\alpha \alpha^{9}\right)}{\left(\alpha \alpha^{3}\right)^{2}} \\
& =a c^{2}+a^{2} c+b^{2} c+b^{2} a-a^{3}-c^{3}, \\
f & =\alpha_{x} \beta_{x}, \\
f_{1} & =\frac{1}{2} \sum_{2} \alpha_{x^{3}} \beta_{x}=a x_{1}^{4}+b x_{1}^{3} x_{2}+b x_{1} x_{2}^{3}+c x_{2}^{4}, \\
L & =\left(x x^{3}\right),
\end{aligned}
$$

* L. E. Dickson, these T r a n s a c tion s, vol. 12 (1911), p. 1, p. 75.

† These Transactions, vol. 14 (1913), pp. 306-310. 


$$
\begin{aligned}
Q & =\frac{\left(x x^{9}\right)}{\left(x x^{3}\right)}=x_{1}^{6}+x_{1}^{4} x_{2}^{2}+x_{1}^{2} x_{2}^{4}+x_{2}^{6}, \\
C_{1} & =\frac{1}{2} \sum_{2}\left(\alpha^{3} \beta\right) \beta_{x}^{2} \\
& =\left(a^{2} b-b^{3}\right) x_{1}^{2}+2\left(a c^{2}-a^{2} c+b^{2} c-b^{2} a\right) x_{1} x_{2}+\left(b^{3}-b c^{2}\right) x_{2}^{2}, \\
C_{2} & =\sum_{2}\left(\alpha \alpha^{9}\right) x^{2}\left(\alpha \alpha^{3}\right)+\alpha_{x}^{2} \beta_{x} q \\
& \left.\left.=(I)+a^{2}\right) x_{1}^{2}-2(a b+b c) x_{1} x_{2}+(l)+c^{2}\right) x_{2}^{2} .
\end{aligned}
$$

It should be noticed that no covariant led by $R$ occurs in this set. In this case it is expressible in terms of the others. In this case $D, a, B$, and $q$ completely characterize the classes (24), but, for $p^{n}>3$, q cannot be substituted for $R$ in characterizing the classes.

The covariant $C_{2}$ is an interesting one because of the fact that the corariants of lowest order led by $D$ and $a^{2}$ alone are respectively

$$
D Q, \quad f^{2},
$$

which are of order six and four respectively: One might expect that $D+a^{2}$ would lead no covariant of order less than twelve. One of this order is

$$
D q^{2}+f^{6} \text {. }
$$

